\title{
Biological characteristics of the A1762T/G1764A mutant strain of hepatitis $B$ virus in vivo
}

\author{
XIAO-HUA LENG ${ }^{1,2^{*}}$, EN-QIANG CHEN ${ }^{1,2^{*}}$, LING-YAO DU $^{1,2}$, LANG BAI $^{1,2}$, \\ DAO-YING GONG ${ }^{3}$, XING CHENG $^{1,2}$, FEI-JUN HUANG ${ }^{3}$ and HONG TANG $^{1,2}$ \\ ${ }^{1}$ Center of Infectious Diseases, West China Hospital, Sichuan University; ${ }^{2}$ Division of Infectious Diseases, \\ State Key Laboratory of Biotherapy, Sichuan University; ${ }^{3}$ Department of Forensic Pathology, \\ Medical School of Basic and Forensic Sciences, Sichuan University, Chengdu, Sichuan 610041, P.R. China
}

Received July 13, 2014; Accepted April 24, 2015

DOI: $10.3892 / \mathrm{mmr} .2015 .4072$

\begin{abstract}
The double nucleotide, A1762T and G1764A exchange (TA mutation), in the hepatitis B virus (HBV) genome basal core promoter $(\mathrm{BCP})$ region is a common viral mutation in patients with chronic HBV infection. This mutation is located in the binding site of hepatocyte nuclear factor 4 (HNF4), and a number of liver-enriched transcription factors are involved in the regulation of HBV transcription and replication. The aim of the present study was to investigate the biological characteristics of the HBV strain with this mutation, and the effect of HNF4 inhibition on the replication of this strain in vivo. The results indicated that in vivo the HBV strain with the TA mutation supported a higher level of pregenomic RNA transcription and HBV DNA replication, compared with the wild-type strain. Furthermore, the concentration of serum HBeAg in the TA mutant group was lower than that in the wild-type strain. Following treatment of the mice with entecavir (ETV) or tenofovir disoproxil fumarate (TDF), the transcription and replication levels of wild-type and mutant strains were reduced. In the groups treated with TDF, the inhibition effect was more marked. In hepatocytes in which HNF4 expression was specifically inhibited, the level of $3.5 \mathrm{~kb}$ mRNA of HBV was reduced compared with that in mouse cells with normal HNF4 expression, and HBV DNA replication levels were also reduced to a greater extent. Furthermore, following liver-specific knockdown of HNF4, the reduction in variant virus expression was greater than that of the wild-type virus. In conclusion, the replication capacity of $\mathrm{HBV}$ with the TA mutation was increased, and the mutation was associated with a reduction in serum $\mathrm{HBeAg}$ levels. This mutant strain
\end{abstract}

Correspondence to: Professor Hong Tang, Center of Infectious Diseases, West China Hospital, Sichuan University, 37 Guo Xue Xiang, Chengdu, Sichuan 610041, P.R. China

E-mail: htang6198@hotmail.com

*Contributed equally

Key words: hepatitis B virus, A1762T/G1764A mutation, hepatocyte nuclear factor 4 , transcription and replication, drug sensitivity remained sensitive to ETV and TDF, and HNF4 supported a higher replication level of TA mutant $\mathrm{HBV}$ in vivo.

\section{Introduction}

Globally, liver disease, which is frequently associated with persistent hepatitis B virus (HBV) infection, remains a significant health problem. More than 350 million people are chronic carriers, despite the existence of effective vaccinations (1). As a member of the hepadnavirus family, HBV has a genome that is a partially-double stranded circular DNA, of $\sim 3.2 \mathrm{~kb}$ in length. The viral genome contains four partially overlapping open reading frames (ORFs), including ORF $\mathrm{P}$, which codes for the polymerase gene; ORF C, for the core gene; ORF S, for the surface or envelope gene; and ORF X, for the regulatory $\mathrm{X}$ gene. The HBV $\mathrm{C}$ gene is divided into two parts by two initiation cordons (ATG); the core region and the pre-core (PC) region (2). The PC region is located upstream of the HBV $\mathrm{C}$ gene. The mRNA transcription from the $\mathrm{PC}$ region is translated into the $\mathrm{HBeAg}$ protein and released into the bloodstream. A proportion of mRNAs transcribed from the core region result in the production of pregenomic RNA, which is wrapped into the core granules and reverse transcribed into viral DNA. The remainder is translated into $\mathrm{HBcAg}$ following splicing. The nucleotide near the $\mathrm{C}$ gene segment is an important component in HBV replication, containing a variety of regulation sequences. The $\mathrm{C}$ gene promoter $(\mathrm{Cp})$ partially overlaps with the 3'-terminus of the $\mathrm{X}$ gene $(\sim n t 1837)$ and the 5'-terminus of the $\mathrm{PC}$ region in the $\mathrm{C}$ gene (nt1838 ). Cp is the most important regulatory factor for $\mathrm{HBV}$ transcription, and is composed of two parts: The core upstream regulatory sequence (CURS, nt1643-1742) and the basal core promoter (BCP, nt1742-1849). The double nucleotide A1762T and G1764A exchange is the most common BCP mutation, and is also termed the TA mutation (3). Clinically, patients with chronic hepatitis B infection, who are serum HBeAg-negative but HBV DNA-positive, often exhibit the TA mutation. A number of reports have shown that the reduction in the $\mathrm{PC}$ region mRNA transcription appears to result in a reduction in $\mathrm{HBeAg}$ expression, while changes in the ability of the transcriptional regulatory factor to bind with the promoter, and the composition of precore and pregenomic RNA, may affect HBV DNA replication (4-6). 
A large number of clinical studies have shown that continuous HBV replication within the body is an important cause of the development and progression of liver disease (7-9). Therefore, antiviral therapy has become the mainstay of treatment for HBV. Interferon $\alpha$ and nucleoside analogues remain the primary treatment options for chronic hepatitis B. A number of clinical studies have shown that, in patients treated with current antivirals for one year, $<30 \%$ had achieved HBeAg seroconversion, and $\sim 70-80 \%$ required long-term antiviral therapy (10). However, treatment with long-term nucleoside analogues may result in the development of drug resistance. The basal core promoter mutation (A1762T and G1764A; TA) is one of the commonest HBV variants, and treatment of this particular strain remains challenging $(11,12)$. Currently, among the five nucleoside analogues approved for chronic hepatitis B treatment, entecavir (ETV) and tenofovir disoproxil fumarate (TDF) exhibit potent efficacy in the inhibition of viral replication and low incidence of resistance, and are thus recommended as first-line agents for the initial treatment of hepatitis B in Europe and the United States. However, the sensitivity of the TA mutation for ETV and TDF remains unclear. To the best of our knowledge there have been no in vivo studies demonstrating their effectiveness in this strain.

In the life cycle of the hepatitis B virus, the 3.5-, 2.4-, 2.1- and $0.7-\mathrm{kb}$ mRNAs are the central transcription products of the viral genome. The pregenomic $3.5-\mathrm{kb}$ mRNA is reverse transcribed to encapsidated viral genomic DNA by the viral polymerase (13). Viral transcription is regulated by numerous host factors. Hepatocyte nuclear factor 4 (HNF4), which contains a zinc finger region and is expressed primarily in the liver, is one of the principal host transcription factors that binds DNA as a dimer and regulates a number of liver-specific genes. HNF4 $\alpha$ is a member of the HNF4 subfamily (14). A gene chip-based study demonstrated that HBV infection increased the transcription of numerous genes, including HNF4 (15). In a recent study by this group, the detection of liver tissue samples from HBV-infected patients indicated that high-expression of HNF4 $\alpha$ may be associated with the occurrence of severe hepatitis B (SHB) (16). Furthermore, clinical data have shown that the TA mutation is related to an increased risk of SHB in patients with chronic HBV infection (17). Therefore, it was hypothesized that there may be an association between the TA mutation and HNF4 $\alpha$ expression. In studies using HepG2 and Huh7 hepatoma cell lines, $\mathrm{HNF} 4 \alpha$ has been shown to be involved in HBV $3.5 \mathrm{~kb}$ pregenomic RNA synthesis and viral replication (18). There is evidence that the TA mutation is located in the proximal nuclear hormone receptor binding site of the core promoter region (3). This suggests that the mutation may exert a degree of influence on binding between HNF4 and the core promoter region. However, the majority of studies on the TA mutation and $\mathrm{HNF} 4 \alpha$ were conducted in vitro, and the association in vivo remains unclear. In the present study, the influence of $\mathrm{HNF} 4 \alpha$ on TA mutant and wild-type HBV transcription and replication was investigated via liver-specific silencing of HNF4 $\alpha$ expression in vivo.

In the current study, a Balb/c mouse model for the replication of the HBV TA mutant was established via a hydrodynamic-based procedure. Using the model and corresponding experimental techniques, the differences in transcription and replication levels between the wild-type and the TA mutant strains were examined. Furthermore, the differences in the effects of ETV, TDF and HNF4 on HBV replication were investigated.

\section{Materials and methods}

Ethics statement. Ethical approval was obtained from the Laboratory Animal ethics committee of Sichuan University (Chengdu, China).

Plasmid. The HBV DNA 4.1-kb plasmid (pHBV4.1 wt) construct is an HBV transcription, replication and expression competent plasmid, which contains 1.3 copies of the HBV genome (subtype ayw) $(19,20)$. The TA mutation, with two nucleotide substitutions (A1762T and G1764A) in the proximal nuclear hormone receptor binding site of the core promoter region, was introduced into the wild-type pHBV4.1 wt via site-directed mutagenesis. The resulting mutant plasmid was named pHBV4.1TAmut. In a previous study by this group, a liver-specific RNA interference (RNAi) plasmid targeting HNF4 $\alpha$ (named pHNF4sh-EP) was successfully constructed and was inserted by directional cloning liver-specific regulatory elements AFPe-ALBp (referred as EP) into the vector plasmid HNF4sh-CMV.pHNF4sh-EP is able to efficiently and liver-specifically silence HNF4 $\alpha$ expression (21).

Mouse model for $\mathrm{HBV}$ replication. Male BALB/C mice, which were purchased from the Huaxi Laboratory Animal Center of Sichuan University, were 18-20 g and 6-8 weeks old, and were maintained under specific-pathogen-free (SPF) conditions. All mice received canonical care under the Institutional Review Board, according to Animal Protection Art of Sichuan University, including keeping mice in a $12 \mathrm{~h}$ light-dark cycle, and at a constant temperature and humidity.

In order to establish the HBV replication mouse model, $10 \mu \mathrm{g}$ (pHBV4.1 wt or pHBV4.1TAmut) plasmids, dissolved in phosphate-buffered saline (PBS), were injected into the mouse tail vein over 5-8 sec (hydrodynamic in vivo transfection) (21). The volume of PBS injected was $10 \%$ of the mouse body weight. Mice were sacrificed by cervical dislocation at $72 \mathrm{~h}$ post-injection, and liver tissues were stored at $-70^{\circ} \mathrm{C}$, prior to DNA and RNA extraction and analysis. Blood samples were allowed to stand overnight at $4^{\circ} \mathrm{C}$, and the serum was then separated and stored at $-20^{\circ} \mathrm{C}$

Inhibition of HNF4 $\alpha$ expression via RNAi. The expression of HNF4 in the liver tissue was specifically inhibited using an HNF4sh-EP plasmid. The pHNF4sh-EP (50 $\mu \mathrm{g})$ dissolved in $2 \mathrm{ml}$ PBS was rapidly injected into mouse tail veins. After 4 days, pHBV4.1wt and pHBV4.1TAmut were injected in the same manner. Mice were sacrificed 3 days later by cervical dislocation. Samples of liver tissues were frozen at $-70^{\circ} \mathrm{C}$ and serum was saved at $-20^{\circ} \mathrm{C}$.

Detection of antiviral effects. $24 \mathrm{~h}$ after the injection of wild-type (pHBV4.1 wt) and TA mutant-type (pHBV4.1TAmut) plasmid, ETV (Bristol-Myers Squibb, New York City, NY, USA; $0.075 \mathrm{mg} / \mathrm{kg} /$ day), TDF (Gilead Sciences, Foster City, CA, USA; $45 \mathrm{mg} / \mathrm{kg} /$ day) or normal saline (control group) were administrated via oral gavage three times at $24 \mathrm{~h}$ intervals. 
The drug dose was determined, based on the recommended human dosage. Mice were sacrificed 4-6 h after the final oral gavage. Liver tissues were conserved as described previously.

Detection of HBV RNA by northern blotting. Frozen mouse liver tissues were mechanically pulverized in liquid nitrogen. Liver tissue powder (0.03-0.05 g) was dissolved in $1 \mathrm{ml} \mathrm{TRIzol}{ }^{\mathrm{TM}}$ reagent (Beijing Solarbio Science and Technology Co., Ltd., Beijing, China). HBV RNA was isolated as described previously (22). After blending, Turbid liquid was extracted twice with chloroform (Sinopharm Chemical Reagent Co., Ltd., Shanghai, China). The supernatant was precipitated with $0.5 \mathrm{ml}$ of isopropanol (Sinopharm Chemical Reagent Co., Ltd.), and resuspended in $100 \mu \mathrm{l}$ of diethylpyrocarbonate water. Using probes for GAPDH and HBV, $30 \mu \mathrm{g}$ HBV RNA was analyzed by northern blotting hybridization, with the GAPDH serving as an internal control. The levels of HBV RNA were calculated using Quantity One software, according to the manufacturer's instructions (Quantity One 1-D version 4.6.2; Bio-Rad Laboratories, Inc., Hercules, CA, USA).

Detection of HBV DNA replication intermediates by southern blotting. Frozen liver tissues were ground to powder in liquid nitrogen, and HBV DNA replication intermediates were isolated from $0.12 \mathrm{~g}$ liver tissue powder, as described previously (21). Viral DNA replication intermediates, resuspended in $30 \mathrm{ul}$ of $10 \mathrm{mmol} / \mathrm{l}$ Tris hydrochloride ( $\mathrm{pH} 8.0)$ and $1 \mathrm{mmol} / \mathrm{l}$ EDTA, were analyzed by southern blotting. Membranes were hybridized with digoxigenin-labeled (Roche Applied Science) HBV ayw genomic DNA (Sigma-Aldrich, St. Louis, MO, USA) in order to detect HBV sequences. HBV sequences were detected by analysis of hybridization of the membranes with digoxigenin-labeled (Roche Applied Science) HBV ayw genomic DNA. The levels of HBV DNA replication intermediates were calculated using Quantity One software, according to the manufacturer's instructions.

Analysis of hepatitis $B$ e antigen $(\mathrm{HBeAg})$ by enzyme-linked immunosorbent assay (ELISA). Three days after the HBV replication mouse model was established, $>50 \mu \mathrm{l}$ serum was collected from each mouse. The levels of HBeAg in mouse sera were measured using $\mathrm{HBeAg}$ detection kits, according to the manufacturer's instructions (Shanghai Shiye Kehua Company, Shanghai, China). Absorbance was measured with dual-wavelength measurement $(450 / 645 \mathrm{~nm})$ on a microtiter plate reader. Results were considered positive if the $\mathrm{OD}_{450}$ was above the cutoff value ( $2.1 \mathrm{x}$ negative control value).

Analysis of HBsAg and HBcAg by immunohistochemistry. The liver tissue in the embedding cassette was fixed in $10 \%$ formaldehyde for $24 \mathrm{~h}$ in order to confirm that the model had been established successfully. The samples were immunohistochemically stained (23). Paraffin sections were deparaffinized, rehydrated and blocked. Sections were incubated with specific antibodies against HBsAg (mouse anti-HBs, monoclonal, 1:100 dilution; cat. no. MA5-13059; Thermo Fisher Scientific, Rockford, IL, USA) and HBcAg (rabbit anti-HBc, monoclonal, 1:150 dilution; cat. no. RB-1413-A; Neomarkers, Inc., Portsmouth, NH, USA). The secondary antibody consisting of polymer-horseradish peroxidase
A

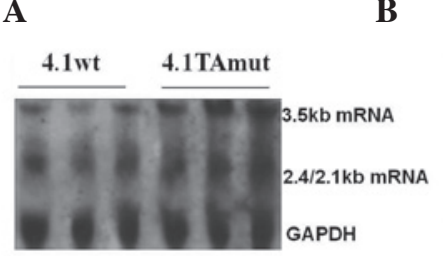

B
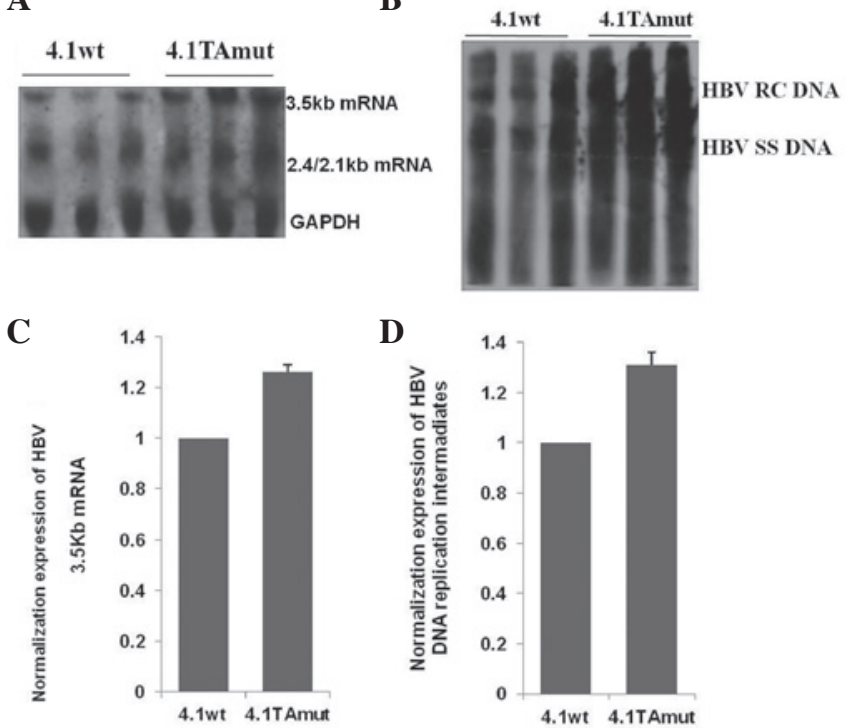

D

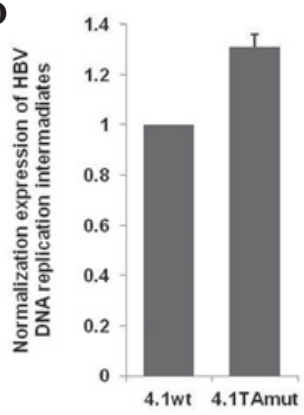

Figure 1. Levels of transcription and replication of HBV $4.1 \mathrm{wt}$ and 4.1TAmut virus in mice liver in vivo. (A) Northern blotting analysis of HBV RNA in mouse liver. GAPDH was used as an internal control. (B) southern blotting analysis of HBV DNA replication intermediates in mouse liver. (C) Quantitative analysis of $3.5 \mathrm{~kb}$ HBV mRNA, according to (A). (D) Relative intensity of HBV DNA replication intermediates. Quantity one software was used for calculation and analysis. The mean value of $4.1 \mathrm{Wt}$ group was defined as 1 . $n=3$. Data are presented as the mean \pm standard deviation from three independent analyses. HBV, hepatitis B virus; RC DNA, relaxed circular DNA; SS DNA, single-stranded DNA; wt, wild-type; mut, mutant

anti-mouse (cat. no. sc65890) or anti-rabbit (cat. no. sc896; Beijing Zhongshan Golden Bridge Biotechnology Co., Ltd., Beijing, China) was applied at 1:350 dilution for $50 \mathrm{~min}$ at $37^{\circ} \mathrm{C}$, according to the manufacturer's instructions. Following counter-staining and finalizing, the liver cells were observed under a microscope (Olympus BX51; Olympus, Tokyo, Japan).

Statistical analysis. Quantity One 1-D analysis software version 4.6.2 was used for calculation and analysis of HBV DNA replication intermediate levels and HBV RNA levels. The data of HBV DNA replication intermediate levels and HBV RNA levels are expressed as the mean \pm standard deviation from three independent experiments using the SPSS software package version 13.0 (SPSS, Inc., Chicago, IL, USA). $\mathrm{P}<0.05$ was considered to indicate a statistically significant difference.

\section{Results}

Biological characteristics of the HBV TA mutant strain in vivo. In order to investigate the transcription and replication characteristics of the HBV TA mutant strain in vivo, HBV RNA and DNA replication intermediate levels were evaluated by northern blotting and southern blotting, respectively (Fig. 1). As shown in Fig. 1A and C, the expression level of the $3.5 \mathrm{~kb}$ HBV mRNA (transcription level) in mouse liver tissue injected with the pHBV4.1TAmut was higher than that of mice injected with pHBV4.1 wt. The expression level of HBV DNA replication intermediates (replication level) of the TA mutant virus was 1.35 -fold higher than that of the wild-type virus (Fig. 1B and D). 
A

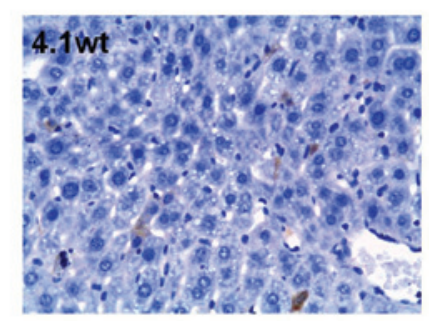

B

C

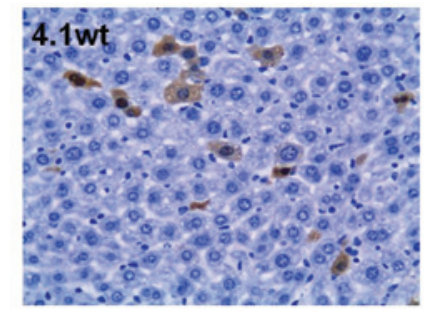

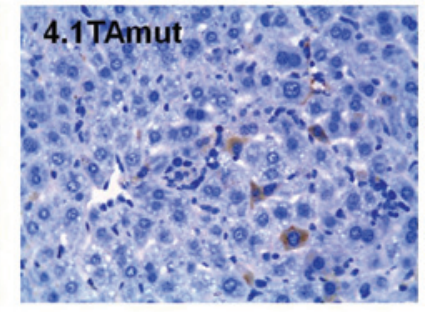

D

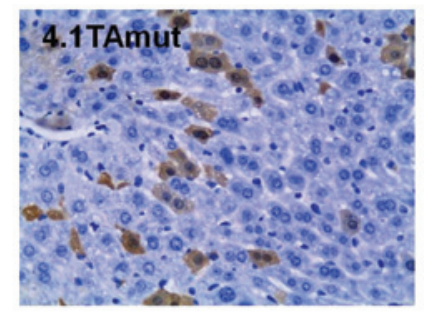

Figure 2. Expression levels of $\mathrm{HBsAg}$ and $\mathrm{HBcAg}$ in mouse liver, detected by immunohistochemistry. (A) and (B) Expression of $\mathrm{HBsAg}$ in pHBV4.1 wt-injected and pHBV4.1TAmut-injected mice; (C) and (D) Expression of HBcAg in pHBV4.1 wt-injected and pHBV4.1TAmut-injected mice. Positive expression is stained brown (magnification, $\mathrm{x} 400$ ). HBsAg, hepatitis B s antigen; wt, wild-type; mut, mutant.

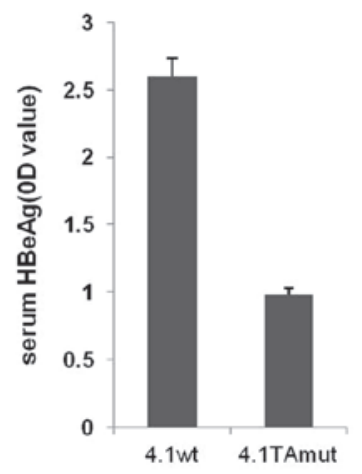

Figure 3. Levels of $\mathrm{HBeAg}$ in mouse serum. Mice were injected with $10 \mu \mathrm{g}$ pHBV4.1 wt or pHBV4.1TAmut. After 3 days, $\mathrm{HBeAg}$ in the serum was measured by ELISA. $n=3$. HBeAg, hepatitis B e antigen; wt, wild-type, mut, mutant; OD, optical density.

As shown in Fig. 2, HBsAg and HBcAg in hepatocytes were stained brown. HBsAg was only detected in the cytoplasm of hepatic cells, whereas HBcAg was detected in the nucleus and the cytoplasm. The number of HBsAg-positive cells in mouse liver tissue injected with pHBV4.1TAmut was greater than that injected with pHBV4.1 wt (Fig. 2A and B). Similarly the number of cells that stained positive for $\mathrm{HBcAg}$ in the cytoplasm and nuclei was greater in cells treated with pHBV4.1TAmut than cells treated with pHBV4.1 wt (Fig. 2C and D). These results suggest that the expression of $\mathrm{HBsAg}$ and $\mathrm{HBcAg}$ from the TA mutant virus was increased compared with that of the wild-type HBV.

Following injection of the different plasmids, the level of HBeAg in mouse serum was detected (Fig. 3). An ELISA assay demonstrated that the level of serum $\mathrm{HBeAg}$ in wild-type mice was higher $(\mathrm{OD}=2.6)$ compared with that in mice injected with the TA-mutant plasmid $(\mathrm{OD}=0.98)$.

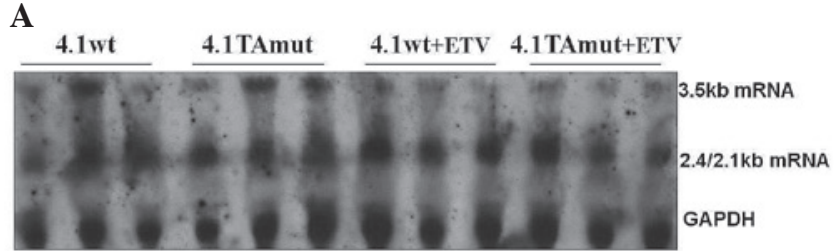

B

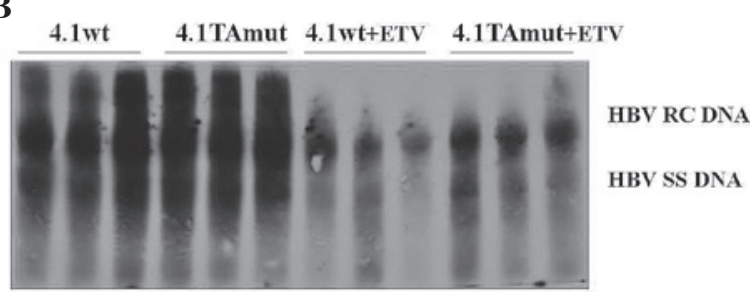

C

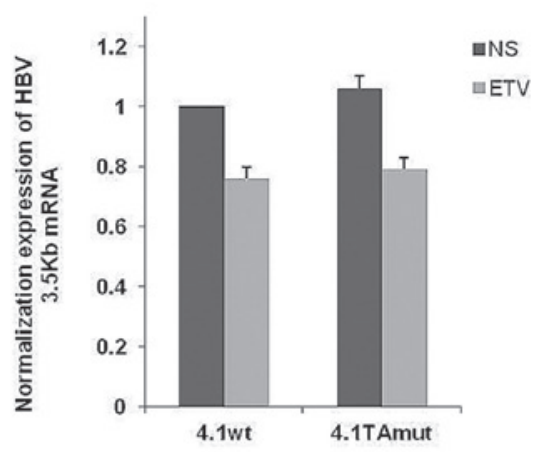

D

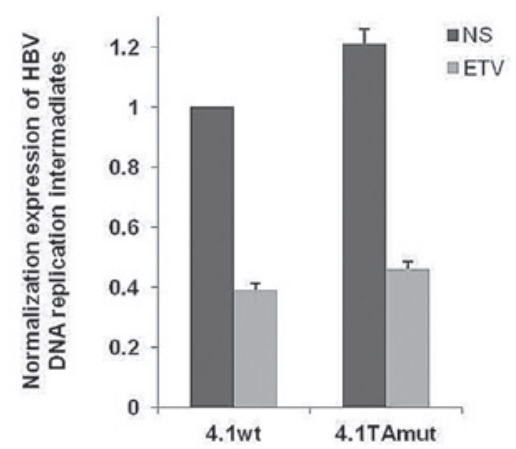

Figure 4. Evaluation of the effect of nucleoside analogue, ETV, on HBV 4.1 wt and 4.1TAmut virus transcription and replication in vivo. (A) Northern blotting analysis of HBV RNA levels in mice treated with NS or ETV. GAPDH was used as an internal control. (B) southern blotting analysis of HBV DNA replication intermediates levels in mice treated with NS or ETV. (C) Quantitative analysis of $3.5 \mathrm{~kb} \mathrm{HBV}$ mRNA according to (A). (D) Relative intensity of HBV DNA replication intermediates. Quantity one software was used for calculation and analysis. The level of $4.1 \mathrm{Wt}$ group was set to 1 . $n=3$. Data are presented as the mean \pm standard deviation from three independent analyses. ETV, entecavir; HBV, hepatitis B virus; NS, normal saline.

The results demonstrated that the TA mutation may have an impact on HBeAg expression, which is consistent with our clinical observation.

Inhibitory effects of antiviral drugs on variant $H B V$. In order to analyze the effect of antiviral drugs, two nucleoside analogues, ETV and TDF, were used to treat mice infected with wild type or variant virus. The level of transcription and 
A

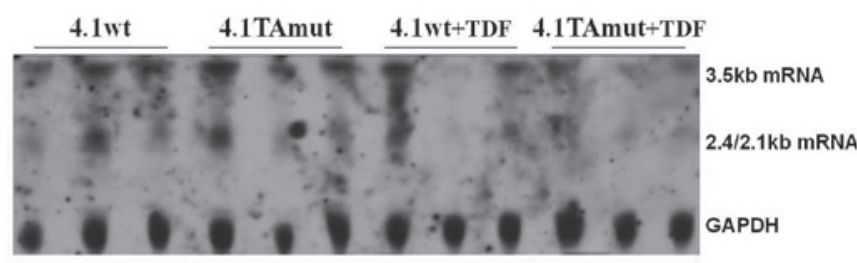

C

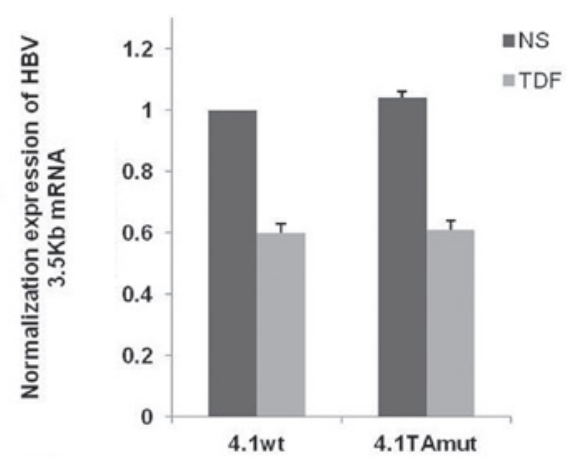

B

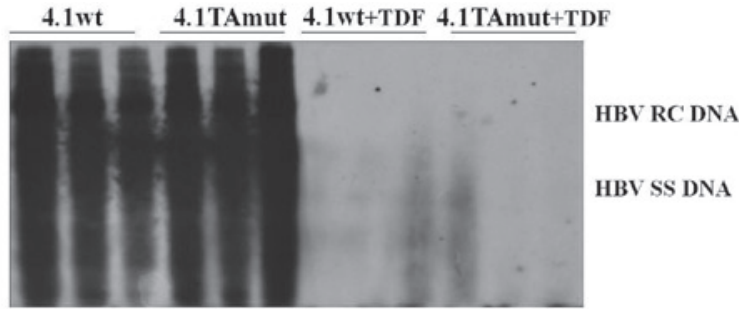

D

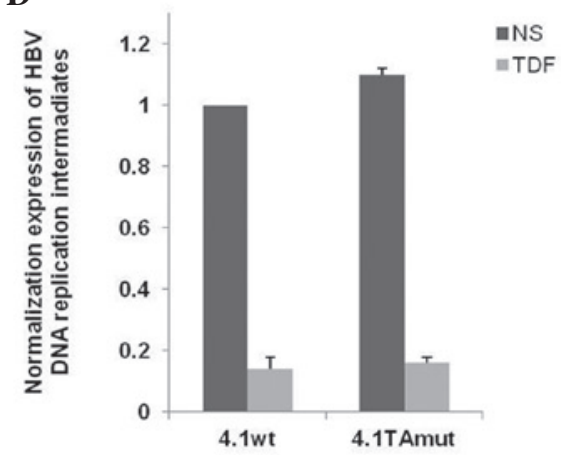

Figure 5. Evaluation of the effect of nucleoside analogue, TDF, on HBV $4.1 \mathrm{wt}$ and 4.1TAmut virus transcription and replication in vivo. (A) Northern blotting analysis of HBV RNA levels in mice treated with NS and TDF. GAPDH was used as an internal control. (B) southern blotting analysis of HBV DNA replication intermediates levels in mice treated with NS and TDF. (C) Quantitative analysis of $3.5 \mathrm{~kb} \mathrm{HBV} \mathrm{mRNA} \mathrm{according} \mathrm{to} \mathrm{(A).} \mathrm{(D)} \mathrm{Relative} \mathrm{intensity} \mathrm{of} \mathrm{HBV} \mathrm{DNA}$ replication intermediates. Quantity one software was used for calculation and analysis. The level of $4.1 \mathrm{Wt}$ group was set to $1 . \mathrm{n}=3$. Data are presented as the mean \pm standard deviation from three independent analyses. TDF, tenofovir disoproxil fumarate; HBV, hepatitis B virus; NS, normal saline.

A

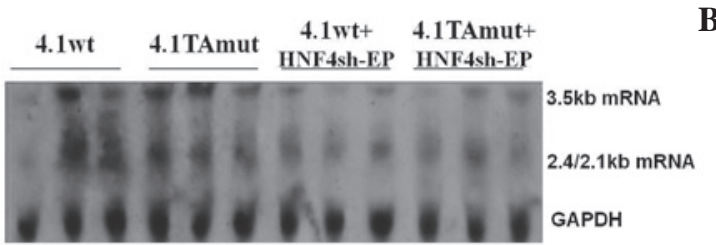

C

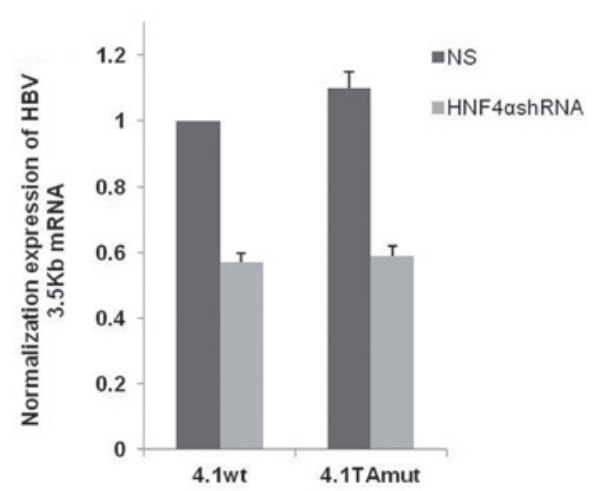

B

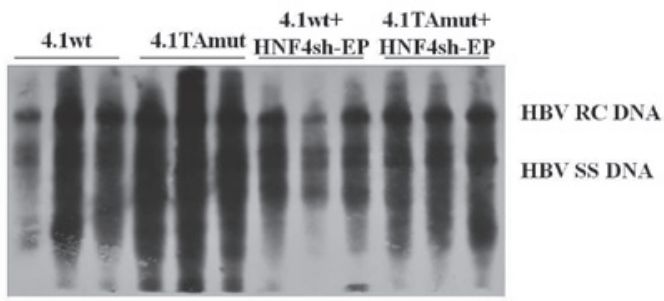

D

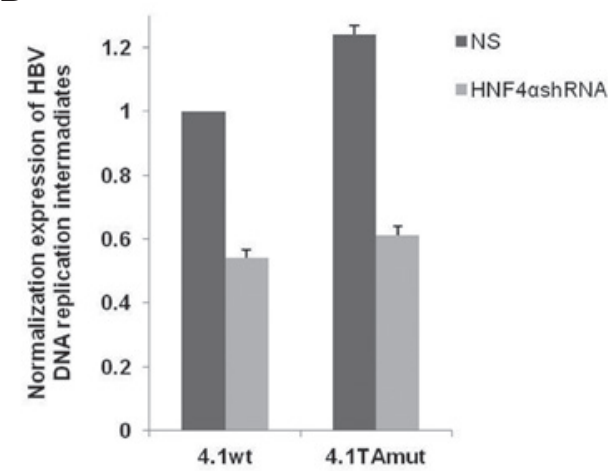

Figure 6. Levels of transcription and replication of HBV $4.1 \mathrm{wt}$ and 4.1TAmut virus following silencing of HNF4 expression in mouse liver in vivo. (A) Northern blotting analysis of HBV RNA in mouse liver. GAPDH was used as an internal control. (B) southern blotting analysis of HBV DNA replication intermediates in mouse liver. (C) Quantitative analysis of $3.5 \mathrm{~kb} \mathrm{HBV}$ mRNA according to (A). (D) Relative intensity of HBV DNA replication intermediates. Quantity one software was used for calculation and analysis. The level of $4.1 \mathrm{Wt}$ group was set to $1 . \mathrm{n}=3$. Data are presented as the mean \pm standard deviation from three independent analyses. HBV, hepatitis B virus; RC DNA, relaxed circular DNA; SS DNA, single-stranded DNA; HNF4, hepatocyte nuclear factor 4.

replication was measured (Figs. 4 and 5). In the ETV-treated group, transcription and replication levels of the wild-type strain were reduced by 24 and $61 \%$, respectively, compared with the control group, and in the TA mutant strain, the transcription and replication levels decreased by 26 and $62 \%$, respectively (Fig. 4). In the TDF-treated group, the transcription and replication levels of the wild-type strain were reduced by $40 \%$ and $86 \%$, respectively, compared with the control group, and the transcription and replication levels in the TA mutant strain decreased by $42 \%$ and $85 \%$, respectively (Fig. 5). The results indicate that the HBV TA mutant strain remains sensitive to ETV and TDF. Furthermore, both 
the strains were more sensitive to TDF than they were to ETV.

Effects on virus transcription and replication following inhibition of HNF4 $\alpha$ expression in vivo. Following transfection with pHNF4sh-EP, which was able to specifically downregulate the expression of HNF4 in the liver, the transcription and replication characteristics of the wild-type and TA mutant-type HBV variants were investigated. The relative levels of virus transcription and replication are presented in Fig. 6. When $\mathrm{HNF} 4 \alpha$ expression was reduced in mouse liver, transcription levels of wild-type and TA mutant virus were decreased by 43 and $47 \%$, respectively (Fig. 6A and C). Similarly, the expression of HBV DNA replication intermediates synthesized from wild-type or TA mutant virus was decreased by 46 and $51 \%$, respectively (Fig. $6 \mathrm{~B}$ and $\mathrm{D}$ ). These results demonstrated that HNF4 exerted a more significant impact on the transcription and replication of the TA mutant HBV.

\section{Discussion}

HBV infection remains a significant global public health issue. Following infection with $\mathrm{HBV}$, hepatitis, liver fibrosis, cirrhosis and liver cancer may occur as the disease progresses $(24,25)$. Each year $\sim 1$ million people die from HBV-related diseases (26). Therefore, the treatment of hepatitis B, and in particular the development of antiviral agents, is of worldwide concern. However, when an anitviral drug is administered for an extended period of time, there is a greater chance of the development of drug-resistant strains of the virus, due to viral mutations. The TA mutation, in which there is an $\mathrm{A} \rightarrow \mathrm{T}$ substitution at nt 1762 and a $\mathrm{G} \rightarrow \mathrm{A}$ mutation at nt 1764 in the $\mathrm{BCP}$ region of $\mathrm{HBV}$, is a common mutation that has been found to confer resistance in clinical trials. The 'immune escape' caused by the reduction in precore protein RNA and $\mathrm{HBeAg}$ synthesis, is an important pathogenic mechanism for the pre-C mutant strains. The TA mutant strain has been reported to be associated with the levels of HBV DNA replication and $\mathrm{HBeAg}$ expression $(27,28)$. The majority of studies on TA mutation have been conducted in cell cultures or in clinical studies. However, few studies have investigated the characteristics of this HBV strain in vivo. Compared with in vitro experiments, studies using appropriate animal models may simulate human diseases more closely. Transgenic mice have been widely used in studies on HBV. The HBV replication mouse model established in this laboratory, restores virus transcription and replication process more authentically (29), and saves time and money, particularly in the study of HBV mutant strains. Using the HBV replication mouse model, our group has investigated the biological characteristics of the TA mutant strain, in addition to the effect of HNF4 on the mutant HBV.

The present results indicated that the replication level of HBV DNA with the TA mutation was increased in mouse liver samples, while the expression level of HBeAg in serum was decreased. A previous in vitro study demonstrated that a higher proportion of pregenomic RNAs from the TA mutant HBV genome were encapsidated, compared with that of the wild-type strain, thus producing higher levels of replication intermediates of variant virus (18). This is in accordance with the results from the present in vitro study. The TA mutation affects the AT-rich regions in the core promoter, which are located upstream of the mRNA start points in viral promoters. These regions are hypothesized to initiate transcription by binding with RNA polymerase (30). The synthesized level of mRNA may influence the replication level of viral DNA. The replication level of the mutant strain may increase following subtle alteration in the levels of pregenomic mRNA transcription and encapsidation. In the present study, the synthesis of $\mathrm{HBeAg}$ in vivo was suppressed, although not fully abolished, in the presence of the TA mutation. HBeAg was synthesized by pre-core mRNA and secreted into the serum. The TA mutation inhibited the synthesis of PC mRNA, thereby reducing the expression of $\mathrm{HBeAg}(4,31)$. Therefore, the TA mutation appears to influence the serum HBeAg level of patients infected with this strain of HBV (32). As an immune tolerant antigen, $\mathrm{HBeAg}$ is involved in the virus persistence in infected individuals (33). For viral variants expressing little or no $\mathrm{HBeAg}$, there is a weaker selection force from the anti-HBe immune response of hosts (6). Certain researchers have postulated that $\mathrm{HBeAg}$ in the serum inhibits $\mathrm{HBV}$ replication, and that viral replication level may increase in conjunction with a decrease in HBeAg levels (30). The TA mutant strain may be more dominant than the wild-type strain in vivo.

Nucleoside analogues, which inhibit HBV replication, are important in antiviral therapy. In recent years, due to advances in the antiviral treatment of chronic hepatitis B (CHB), international associations on liver diseases have updated their guidelines on the management of $\mathrm{CHB}$, to emphasize that the goal of CHB antiviral treatment is to induce maximal long-term suppression of viral replication, thereby delaying the progression and occurrence of cirrhosis, hepatocellular carcinoma and liver failure. However, following a period of continuous treatment, virological breakthrough may occur, primarily as a result of the development of drug resistance. Drug resistance of $\mathrm{HBV}$ is predominantly due to mutations in the $\mathrm{P}$ region of the HBV genome. However, the sensitivity to nucleoside analogues merited further research in strains with mutation in other regions. In the multidrug-resistant variant, rtA181 V/T, for example, the drug sensitivity to lamivudine (LAM), adefovir dipivoxil (ADV), and TDF was downregulated by a factor of $10,2-8$ and 2-3, respectively, although the sensitivity of this strain to ETV was undiminished (34). LAM therapy resulted in the rapid development of TA mutants in HBeAg-positive patients during one clinical trial (35). An in vitro study demonstrated that the TA mutant strain remained resistant to LAM, while it was sensitive to ADV (2). ETV is a cyclopentanoyloxy guanosine analogue and TDF is a single adenosine analogue. Clinical trials have shown that ETV and TDF are safe and effective for long-term use in patients with CHB (36). The 2012 guidelines published by the Asian Pacific Association for the Study of the Liver, recommended ETV and TDF as the nucleoside analogues of choice. However for viruses with the TA mutation, the antiviral effect of ETV and TDF is unclear. In the present study, ETV and TDF were administrated via oral gavage three times. The results showed that they were similarly effective in wild-type compared with TA mutant mice models, while TDF showed greater antiviral efficacy in each strain.. 
Liver enriched transcription factor is a class of protein molecule with gene transcription regulation function. A number of forms of liver enriched transcription factors, such as hepatocyte nuclear factor 4 (HNF4), HNF1, retinoid X receptor $\alpha(\mathrm{RXR} \alpha)$ and peroxisome proliferator-activated receptor $\alpha(\operatorname{PPAR} \alpha)$ heterodimers, are able to bind with four promoters of the HBV genome, and are involved in regulating HBV gene transcription and viral replication (37). A previous study showed that HNF4 and RXR $\alpha$-PPAR $\alpha$ heterodimers activate the transcription of the HBV core promoter (18). It may be that these nuclear hormone receptors, which are primarily expressed in hepatocytes, may restrict viral mRNA transcription and DNA synthesis. Synthesis of pregenomic $3.5-\mathrm{kb}$ mRNA begins from the nucleocapsid promoter of $\mathrm{C}$ region. The nuclear hormone receptor binding site is located in the nucleocapsid promoter and encompasses the location of the TA mutation, with the two nucleotide substitutions of $\mathrm{A} \rightarrow \mathrm{T}$ at nt 1762 and $\mathrm{G} \rightarrow \mathrm{A}$ at nt 1764 (18). Previous analysis has indicated that the binding properties of transcription factors are altered in the nuclear hormone receptor binding site of the variant virus. RXR $\alpha$-PPAR $\alpha$ and HNF4 may bind to the nuclear hormone receptor binding sites in the wild-type nucleocapsid promoter and activate transcription. By contrast, the variant nuclear hormone receptor sites combined with HNF4 but not RXR $\alpha$-PPAR $\alpha$ (27). In the current study, pHNF4sh-EP was transfected into the mice via hydrodynamic-based injection, in order to knock down HNF4. As the TA mutation blocked the binding of the RXR $\alpha$-PPAR $\alpha$ heterodimers with the promoter, the variant viral gene expression was regulated only by HNF4. Therefore, silencing of HNF4 resulted in a significant reduction in transcription and replication of the mutant strain. The effect of specific nuclear hormone receptors on pregenomic RNA synthesis and viral replication, indicated that RXR $\alpha$-PPAR $\alpha$ in liver cells, supports high levels of transcription and replication of wild-type strain, while HNF4 supports high levels of transcription and replication of the TA mutation strain.

In conclusion, the present study provided further evidence that the double nucleotide substitutions (A1762T and G1764A) in the $\mathrm{BCP}$ region of the hepatitis $\mathrm{B}$ virus may increase transcription and replication of this virus, and reduce the level of serum HBeAg patients with HBV infection. ETV and TDF therapy were found to be effective in decreasing levels of wild-type and TA mutant-type HBV, which may be significant in clinical practice. In addition, a high level of HNF4 promoted TA mutant HBV replication in vivo.

\section{Acknowledgements}

This study was supported by grants from the National Natural Science Foundation of China (grant no. 81271811) and National Science and Technology Major Project of China (grant no. 2012ZX10002007-001-003). The authors would like to thank Alan McLachlan (Department of Microbiology and Immunology, College of Medicine, University of Illinois at Chicago, IL, USA) for plasmid, pHBV4.1.

\section{References}

1. Dandri M,Lutgehetmann M and Petersen J: Experimental models and therapeutic approaches for HBV. Semin Immunopathol 35: $7-21,2013$
2. Tacke F, Gehrke C, Luedde T, Heim A, Manns MP and Trautwein C: Basal core promoter and precore mutations in the hepatitis B virus genome enhance replication efficacy of Lamivudine-resistant mutants. J Virol 78: 8524-8535, 2004.

3. Gunther S, Piwon N and Will H: Wild-type levels of pregenomic RNA and replication but reduced pre-C RNA and e-antigen synthesis of hepatitis B virus with C $(1653) \rightarrow \mathrm{T}, \mathrm{A}(1762) \rightarrow \mathrm{T}$ and $\mathrm{G}(1764) \rightarrow$ A mutations in the core promoter. J Gen Virol 79: 375-380, 1998

4. Laras A, Koskinas J and Hadziyannis SJ: In vivo suppression of precore mRNA synthesis is associated with mutations in the hepatitis B virus core promoter. Virology 295: 86-96, 2002.

5. Li J, Buckwold VE, Hon MW and Ou JH: Mechanism of suppression of hepatitis B virus precore RNA transcription by a frequent double mutation. J Virol 73: 1239-1244, 1999.

6. Parekh S, Zoulim F, Ahn SH, et al: Genome replication, virion secretion and e antigen expression of naturally occurring hepatitis B virus core promoter mutants. J Virol 77: 6601-6612, 2003.

7. Nelson PK, Mathers BM, Cowie B, et al: Global epidemiology of hepatitis B and hepatitis $\mathrm{C}$ in people who inject drugs: results of systematic reviews. Lancet 378: 571-583, 2011.

8. Lavanchy D: Hepatitis B virus epidemiology, disease burden, treatment and current and emerging prevention and control measures. J Viral Hepat 11: 97-107, 2004.

9. Maynard JE: Hepatitis B: Global importance and need for control. Vaccine 8 (Suppl): S18-S23, 1990.

10. Tseng TC, Liu CJ, Chen CL, et al: Serum hepatitis B virus-DNA levels correlate with long-term adverse outcomes in spontaneous hepatitis B e antigen seroconverters. J Infect Dis 205: 54-63, 2012.

11. Chu CJ, Keeffe EB, Han SH, et al: Prevalence of HBV precore/core promoter variants in the United States. Hepatology 38: 619-628, 2003.

12. Ouneissa R, Bahri O, Alaya-Bouafif NB, et al: Frequency and clinical significance of core promoter and precore region mutations in Tunisian patients infected chronically with hepatitis B. J Med Virol 84: 1719-1726, 2012.

13. Will H, Reiser W, Weimer T, et al: Replication strategy of human hepatitis B virus. J Virol 61: 904-911, 1987.

14. Sladek FM, Zhong WM, Lai E and Darnell JE Jr: Liver-enriched transcription factor HNF-4 is a novel member of the steroid hormone receptor superfamily. Genes Dev 4: 2353-2365, 1990.

15. Wang Z, Bishop EP and Burke PA: Expression profile analysis of the inflammatory response regulated by hepatocyte nuclear factor $4 \alpha$. BMC genomics 12: 128, 2011.

16. Chen EQ, Sun H, Feng P, et al: Study of the expression levels of Hepatocyte nuclear factor 4 alpha and 3 beta in patients with different outcome of HBV infection. Virol J 9: 23, 2012.

17. Xu L, Chen EQ, Lei J, et al: Pre-core/basal-core promoter and reverse transcriptase mutations in chronic HBV infected-patients. Hepatogastroenterology 59: 212-215, 2012.

18. Buckwold VE, Xu Z, Chen M, Yen TS and Ou JH: Effects of a naturally occurring mutation in the hepatitis B virus basal core promoter on precore gene expression and viral replication. J Virol 70: 5845-5851, 1996.

19. Liu FJ, Liu L, He F, et al: Establishment and primary application of a mouse model with hepatitis B virus replication. World $\mathrm{J}$ Gastroenterol 13: 5324-5330, 2007.

20. Tang $\mathrm{H}$ and McLachlan A: A pregenomic RNA sequence adjacent to DR1 and complementary to epsilon influences hepatitis B virus replication efficiency. Virology 303: 199-210, 2002.

21. Liu F, Song Y and Liu D: Hydrodynamics-based transfection in animals by systemic administration of plasmid DNA. Gene Ther 6: 1258-1266, 1999.

22. Tang H, Delgermaa L, Huang F, et al: The transcriptional transactivation function of HBx protein is important for its augmentation role in hepatitis B virus replication. J Virol 79: 5548-5556, 2005.

23. Chen EQ, Gong DY, Leng XH, et al: Inhibiting the expression of hepatocyte nuclear factor 4 alpha attenuates lipopolysaccharide/D-galactosamine-induced fulminant hepatic failure in mice. Hepatobiliary Pancreat Dis Int 11: 624-629, 2012

24. Ganem D and Prince AM: Hepatitis B virus infection-natural history and clinical consequences. N Engl J Med 350: 1118-1129, 2004.

25. Sumi H, Yokosuka O, Seki N, et al: Influence of hepatitis B virus genotypes on the progression of chronic type B liver disease. Hepatology 37: 19-26, 2003. 
26. Pungpapong S, Kim WR and Poterucha JJ: Natural history of hepatitis B virus infection: An update for clinicians. Mayo Clin Proc 82: 967-975, 2007.

27. Tang H, Raney AK and McLachlan A: Replication of the wild type and a natural hepatitis $\mathrm{B}$ virus nucleocapsid promoter variant is differentially regulated by nuclear hormone receptors in cell culture. J Virol 75: 8937-8948, 2001.

28. Yu H, Zhu R, Zhu YZ, Chen Q and Zhu HG: Effects of mutations in the $\mathrm{X}$ gene of hepatitis $\mathrm{B}$ virus on the virus replication. Acta virologica 56: 101-110,2012.

29. Kim do Y, Kim HJ, Lee CK, et al: Efficacy of adefovir dipivoxil in the treatment of lamivudine-resistant hepatitis B virus genotype $\mathrm{C}$ infection. Liver Int 27: 47-53, 2007.

30. Okamoto H, Tsuda F, Akahane Y, et al: Hepatitis B virus with mutations in the core promoter for an e antigen-negative phenotype in carriers with antibody to e antigen. J Virol 68: 8102-8110, 1994.

31. Scaglioni PP, Melegari M and Wands JR: Biologic properties of hepatitis B viral genomes with mutations in the precore promoter and precore open reading frame. Virology 233: 374-381, 1997.
32. Kim DW, Lee SA, Hwang ES, Kook YH and Kim BJ: Naturally occurring precore/core region mutations of hepatitis B virus genotype $\mathrm{C}$ related to hepatocellular carcinoma. PloS one 7: e47372, 2012.

33. Seeger C and Mason WS: Hepatitis B virus biology. Microbiol Mol Biol Rev 64: 51-68, 2000.

34. Dai J, Chen EQ, Bai L, et al: Biological characteristics of the rtA181T/sW172* mutant strain of Hepatitis B virus in animal model. Virol J 9: 280, 2012.

35. Selabe SG, Song E, Burnett RJ and Mphahlele MJ: Frequent detection of hepatitis B virus variants associated with lamivudine resistance in treated South African patients infected chronically with different HBV genotypes. J Med Virol 81: 996-1001, 2009.

36. Koklu S, Tuna Y, Gulsen MT, et al: Long-term efficacy and safety of lamivudine, entecavir and tenofovir for treatment of hepatitis B virus-related cirrhosis. Clin Gastroenterol Hepatol 11: 88-94, 2013.

37. Su W and T H: Regulation of hepatitis B virus transcription and replication by liver-enriched transcriptional factors. World Chinese Journal of Digestology 15: 1237-1240, 2007. 